\title{
Comunicação de más notícias na educação médica e confluências com o contexto da pandemia de covid-19
}

\section{Delivering bad news in medical education and confluences with the context of covid-19 pandemic}

\author{
Kelen Gomes Ribeiro ${ }^{a}$ \\ (D) https://orcid.org/0000-0003-0870-1971 \\ Email: kelengomesrळyahoo.com.br \\ Maxmiria Holanda Batista ${ }^{a}$ \\ (D) https://orcid.org/0000-0002-9069-678X \\ E-mail: maxmiriaholandaळyahoo.com.br \\ Daiana Flávia Oliveira de Souzab \\ (i) https://orcid.org/0000-0002-3188-7228 \\ E-mail: daianaflaviatı23ufcœoutlook.com \\ Caroline Mary Gurgel Dias Florêncio ${ }^{a}$ \\ (D) https://orcid.org/0000-0002-9297-2856 \\ E-mail: caroline.gurgelळufc.br

\section{Walker Henrique Almeida Jorge \\ (D) https://orcid.org/0000-0001-9667-5229 \\ E-mail: henriquewalkerळgmail.com \\ Cheila Pires Raquel ${ }^{a}$ \\ (iD) https://orcid.org/0000-0002-7463-5450 \\ E-mail: sheilaraquelløgmail.com} \\ anniversidade Federal do Ceará. Faculdade de Medicina. \\ Departamento de Saúde Comunitária. Fortaleza, CE, Brasil. \\ bUniversidade Federal do Ceará. Faculdade de Medicina. \\ Fortaleza, CE, Brasil. \\ 'Universidade Federal do Ceará. Centro de Humanidades II. \\ Departamento de Psicologia. Fortaleza, CE, Brasil.
}

\section{Correspondência}

Kelen Gomes Ribeiro

Departamento de Saúde Comunitária.

Rua Papi Júnior, 1223, 5 andar, Rodolfo Teófilo, Fortaleza, Ceará, Brasil

\section{Resumo}

O artigo apresenta um estudo exploratório descritivo sobre habilidades de Comunicação de Más Notícias (CMN) de internos de Medicina. Investigou-se a percepção discente sobre aprendizagem para CMN com delineamento transversal e abordagem mista. Houve aplicação de questionário, obtendo 176 participantes, e grupo focal com 12 estudantes. Os dados foram analisados pelo cálculo das médias e desvios-padrão para variáveis quantitativas e as falas do grupo focal foram submetidas à Análise de Conteúdo. Observou-se o dobro da frequência com mais habilidade para CMN no grupo que teve treinamento em comparação ao que não teve. No manejo da CMN, considerou-se mais difícil "ser honesto, sem tirar a esperança" (69\%) e "lidar com a emoção do paciente" (59\%). $99,4 \%$ conheciam o protocolo SPIKES, destes 41,5\% consideraram a expressão de emoções sua etapa mais difícil. A comunicação e o manejo das emoções foram apontados como desafios na relação médico-paciente, com déficits no ensino de CMN. Obteve-se que a CMN não se limita à técnica, mas envolve atitudes que precisam ser abordadas com metodologias diversas, assim como requerem a implementação de políticas de educação na área médica, sobretudo diante das demandas emergentes da pandemia de Covid-19.

Palavras-chave: Educação Médica; Comunicação; Relações Médico-Paciente; Educação de Graduação em Medicina. 


\section{Introdução}

The article presents an exploratory, descriptive study on Bad News Communication skills (CMN) of medical interns. The student's perception was on learning for the CMN was investigated with a cross-sectional design and a mixed approach. A questionnaire was applied, 176 participants were obtained, and a focus group was held with 12 students. The data were analyzed through the calculation of means and standard deviations for quantitative variables and the focus group reports were subjected to Content Analysis. It was observed that in the group that had training, compared to the one who did not, ocurred twice frequency with more skill for CMN. In the CMN's management, it was considered harder to be "honest without taking away hope" (69\%) and "to deal with the patient's emotion" (59\%). 99.4\% knew the SPIKES protocol, of which $41.5 \%$ considered the expression of emotions as its most difficult stage. Communication and handling of emotions were pointed out as the main difficulties in the doctorpatient relationship, with deficits in the teaching of CMN. It was found that CMN is not limited to the technical issue, but it involves attitudes that need to be addressed with different methodologies, as well as the implementation of educational policies in the medical field, especially given the demands that emerges with the covid-19 pandemic.

Keywords: Medical Education; Communication; Physician-Patient Relations; Education Medical Undergraduate.
A comunicação é entendida como competência profissional que, por meio de utilização de linguagem compreensível no processo terapêutico, estimula o relato espontâneo da pessoa sob cuidados, considerando os aspectos psicológicos, culturais e contextuais, sua história de vida, o ambiente em que vive e suas relações sociofamiliares, assegurando a privacidade e o conforto. Sua efetividade é muito importante para o desenvolvimento da relação entre médico e paciente, possibilitando uma interação adequada, com condição de conciliar a necessidade de apresentação de um raciocínio clínico com os aspectos psicossociais, emocionais e espirituais de ambos os envolvidos (Brasil, 2014; Oliveira, 2018).

A “má notícia” é definida como informação que carrega enorme valência emocional e tem o potencial de mudar a vida e a perspectiva de futuro das pessoas. Na área da saúde, tradicionalmente, os exemplos de comunicação de más notícias (CMN) passam pelo diagnóstico de doenças ameaçadoras de vida, amputação de membros, comunicação sobre a morte de um familiar. Contemporaneamente, além das citadas, incluemse as doenças que interferem na qualidade de vida (Camargo et al., 2019; Vogel et al., 2019).

A CMN, embora seja frequente no exercício da medicina, ainda é considerada uma tarefa extremamente difícil. Até os anos 1970, era comum não se informar diagnósticos, como o de câncer, dentro de uma consideração de que a notícia poderia levar os pacientes à falta de esperança e de adesão ao tratamento. Os estudos mostraram a importância dessa revelação, mesmo que não seja tarefa simples para nenhum dos envolvidos no processo. A partir dos anos 1980 foram criados protocolos com a finalidade de treinamento e adequação da CMN por parte dos profissionais. Tornaram-se referências para uma prática clínica satisfatória, que considera a subjetividade humana e sua multiplicidade de expressões, também, nos contextos de adoecimento e morte (Stovall, 2015).

O protocolo mais conhecido é o Spikes, que recomenda passos para a CMN. É recomendado como guia para o treinamento das habilidades de 
comunicação, apesar de sofrer algumas críticas. É um mnemônico de seis passos que pode proporcionar mais segurança ao médico e que apresenta quatro objetivos principais: saber o que o paciente e seus familiares estão entendendo da situação como um todo; fornecer as informações de acordo com o que o paciente e sua família suportam ouvir; acolher qualquer reação que pode vir a acontecer e, por último, ter um plano (Cruz et al., 2016).

Mesmo com a existência de ferramentas importantes de CMN como o Spikes e, no caso brasileiro, de um enfoque de priorização da competência comunicacional nas diretrizes curriculares nacionais do curso de graduação em medicina (Brasil, 2014), é possível que o processo didático utilizado para $\mathrm{CMN}$ ao paciente e seus familiares não esteja adequadamente ministrado durante a formação médica.

$\mathrm{Na}$ contemporaneidade, em que se vivencia a maior emergência de saúde pública que a comunidade internacional enfrenta neste século, a pandemia de covid-19, evidencia-se com mais força a necessidade de que a educação médica priorize a efetiva e adequada CMN. Estudo publicado pelo Imperial College analisou a taxa de transmissão ativa da covid-19 em 48 países e demonstrou que o Brasil chegou a apresentar a maior taxa de transmissão (Ro) de 2,81. Quando a redação do presente artigo foi concluída, os números de casos confirmados e de mortos no Brasil ultrapassavam, respectivamente, 5,9 milhões de casos e $168 \mathrm{mil}$ óbitos (Brasil, 2020b).

Os dados apontam para uma alta frequência também no ato de comunicar más notícias. 0 referido ato requer uma postura respeitosa às diferenças individuais e dos grupos, e deve ser adequado a cada pessoa e sua família de forma cuidadosa. Os estudantes de medicina são a esperança de futuro para uma atuação ética, justa e empática (Tang et al., 2013). Neste contexto, com o propósito de identificar as fragilidades e potências na CMN ainda na fase de graduação, o presente estudo tem como objetivo compreender o processo de aprendizagem da CMN a partir da ótica de alunos internos do curso de medicina de uma universidade federal brasileira, apontando reflexões teóricas relacionadas com o contexto da pandemia de covid-19.

\section{Materiais e métodos}

Trata-se de um estudo exploratório e descritivo, com delineamento transversal e abordagem mista, conduzido no período de outubro de 2019 a abril de 2020, coincidindo, no último período, com o início da pandemia no Brasil. Consiste num recorte da pesquisa Comunicação de más notícias no ensino médico. O curso de medicina da Universidade Federal do Ceará (UFC), localizado no campus do Porangabuçu, em Fortaleza, foi selecionado por ser uma das grandes referências para a formação médica no estado, o qual conta com oito escolas médicas, sendo quatro públicas e quatro privadas (Pinheiro, 2017).

A população constituiu-se do total de internos matriculados no curso de medicina da UFC, estudantes que cursam os quatro últimos semestres da graduação em medicina, nomeada de internato. Correspondem a 320 estudantes que têm sua formação médica embasada no currículo proposto a partir de 2001, dado que o atual projeto pedagógico entrou em vigor a partir do primeiro semestre de 2018 e, por isso, ainda não conta com estudantes no internato. Adotaram-se os seguintes critérios de inclusão: ser interno do curso de medicina da UFC, com idade mínima de 18 anos, que aceitasse participar voluntariamente da pesquisa. Foram excluídos aqueles que estavam fora das atividades letivas por estarem de licença doença ou maternidade, e os que já tinham cursado disciplinas da graduação em medicina em outra faculdade.

Não foi realizada nenhuma técnica de amostragem, pois todos os estudantes do internato foram convidados a participar do estudo. Sendo assim, 198 questionários foram recebidos e 176 considerados válidos para análise, pois 17 desses alunos cursaram parte de sua graduação em medicina em outra universidade e 5 respostas estavam duplicadas. Dessa forma, ficaram as respostas validadas distribuídas por semestres do internato: 57 do primeiro semestre, 35 do segundo, 42 do terceiro e 42 do quarto.

A pesquisa foi submetida à Plataforma Brasil, tendo sido analisada e obtido aprovação do Comitê de Ética da Universidade Federal do Ceará (UFC), por meio do parecer de número 3·319·315. 


\section{Estudo quantitativo}

Anterior à análise descritiva, prosseguiu-se a verificação da consistência em busca de possíveis equívocos durante preenchimento do banco. Cada variável foi avaliada criteriosamente. Para as variáveis quantitativas foram realizadas as médias com seus respectivos desvios-padrão (DP). A variável idade foi categorizada em faixas etárias. A variável semestre recebeu categorização I1, I2, I3 e I4, referente aos quatro semestres do internato. Para as variáveis qualitativas foram calculadas as frequências absolutas e relativas (análise univariada). Foram confeccionados gráficos em barras e colunas selecionados de acordo com a melhor representação para aquela variável.

Foram calculadas as razões de prevalência (RP) em relação aos grupos segundo o semestre (S) que estão cursando. Foi escolhida como variável desfecho "a tarefa mais difícil na CMN". A análise bivariada foi realizada através do teste qui-quadrado de Pearson ou de Fischer quando adequado. Foram considerados significativos os valores de $\mathrm{p}$ inferiores a 0,05 (p-valor<5\%) e com intervalo de confiança (IC) de 95\%.

A variável "treinamento" foi dicotomizada em "sim" para aqueles que afirmaram realizar algumas das atividades como acompanhamento de profissional, aula teórica e laboratório de habilidades; e em "não" para aqueles que afirmaram que não realizaram nenhuma das atividades listadas acima. A variável "habilidade" também foi dicotomizada em "sim" para as respostas ditas como "boa" ou "muito boa" e "não" para os que afirmaram ser "ruim" ou "muito ruim".

\section{Estudo qualitativo}

Para o estudo qualitativo, contou-se com a participação voluntária de 12 estudantes no grupo focal (GF), sendo três de cada um dos quatro semestres do internato, que atenderam ao chamado para comparecimento no dia e local propostos. Não houve seleção dos voluntários, mas um convite através dos meios de divulgação da faculdade. No local marcado para o GF, verificou-se se os três primeiros que chegaram de cada semestre atendiam aos critérios de inclusão estabelecidos, visando compor um grupo com representatividade dos quatro semestres do internato e com até 12 participantes, conforme preconizado (Flick, 2009). Consideraram-se as respostas do GF como um todo, mas especificando, ao final da fala registrada, o semestre do participante como I1 a I4, de acordo com o semestre do internado que o estudante cursava no momento da realização do grupo.

O GF foi gravado, transcrito e analisado por meio da análise de conteúdo, com a técnica de análise temática (Bardin, 2011). Os resultados foram apresentados com os temas definidos previamente e expressos nas perguntas do roteiro, como: a educação médica para a má notícia, despreparo emocional para dar más notícias; a percepção sobre o protocolo Spike, e sugestões para aperfeiçoar o desenvolvimento da competência comunicacional. Além desses temas, foram definidos outros dois a posteriori, a partir do trabalho criterioso de análise dos dados: a "terceirização" das más notícias, o poder biomédico e a interdisciplinaridade na má notícia. Os temas centrais do estudo foram a percepção sobre o protocolo Spike e despreparo emocional para dar más notícias.

\section{Resultados}

Um total de 176 estudantes compuseram a amostra. A média de idade foi de 24,3 anos com DP de 3,04, com a mínima de 20 e a máxima de 41 anos. Prevaleceu na amostra o sexo masculino (52\%), pessoas solteiras (95\%), católicas (39\%) ou sem religião (36\%). Não foi observada diferença entre sexo e a escolha na tarefa de más notícias ( $p=0.64$ ). Assim como sexo e habilidade em dar más notícias também não apresentou significância $(\mathrm{p}=0.52$; $\mathrm{RP}=0.92$; IC 0.72 a 1,17), ainda assim foi percebido que os estudantes do sexo masculino se consideram com mais habilidade do que as de sexo feminino.

Em se tratando do semestre no internato, contouse com a participação de estudantes dos semestres 1 - I1 (32,3\%), semestre 2 - I2 (19,9\%), semestre 3 - I3 e semestre 4 - I4 $(23,9 \%$, cada).

A tarefa mais difícil na CMN, para $38 \%$ dos participantes, é conversar sobre o fim do tratamento e para $28 \%$, é comunicar sobre a morte de um paciente a seus familiares. Foram consideradas, 
também, as tarefas de informar diagnóstico de doença grave ( $15 \%$ ), dialogar com o paciente sobre a morte (13\%) e contar sobre a recorrência da doença (6\%). Ao comparar as respostas dos internos, por semestre, as duas atividades consideradas mais difíceis prevaleceram dos semestres 1 ao 4 .

0 grupo de estudantes que realizou treinamento possui o dobro da frequência a mais com habilidade para contar más notícias do que o grupo que não realizou ( $p=0.000$; IC 1.44 a 2.83; RP=2,02). Não foi observada relação entre o semestre do acadêmico e a tarefa que considerava mais difícil para comentar com o paciente $(\mathrm{p}=\mathrm{o} .81)$. Existe diferença estatística entre os semestres e a habilidade em contar más notícias ( $\mathrm{p}=\mathrm{o}$.0oo). 0 primeiro semestre apresentou sempre menos habilidade quando comparado aos outros semestres ( $p=0.000$; IC=0.33 a o.69; RP o.47). O segundo semestre não apresentou diferença entre os grupos que afirmam ter ou não habilidade ( $\mathrm{p}=\mathrm{0.50}$; $\mathrm{IC}=0,87$ a 1.45; $\mathrm{RP}=1.10$ ). 0 terceiro semestre se mostrou com maior frequência com significância estatística ( $p=0,047$; IC=1.03 A 1.64; RP=1.30), assim como o quarto semestre também se mostrou significativo para este desfecho $(\mathrm{p}=\mathrm{o}, \mathrm{o} 6 ; \mathrm{IC}=1.14 \mathrm{a}$ 1.77; RP=1.42), sendo que a habilidade foi observada em $40 \%$ a mais neste grupo.

\section{Gráfico I - Tarefa mais difícil na comunicação de más notícias}

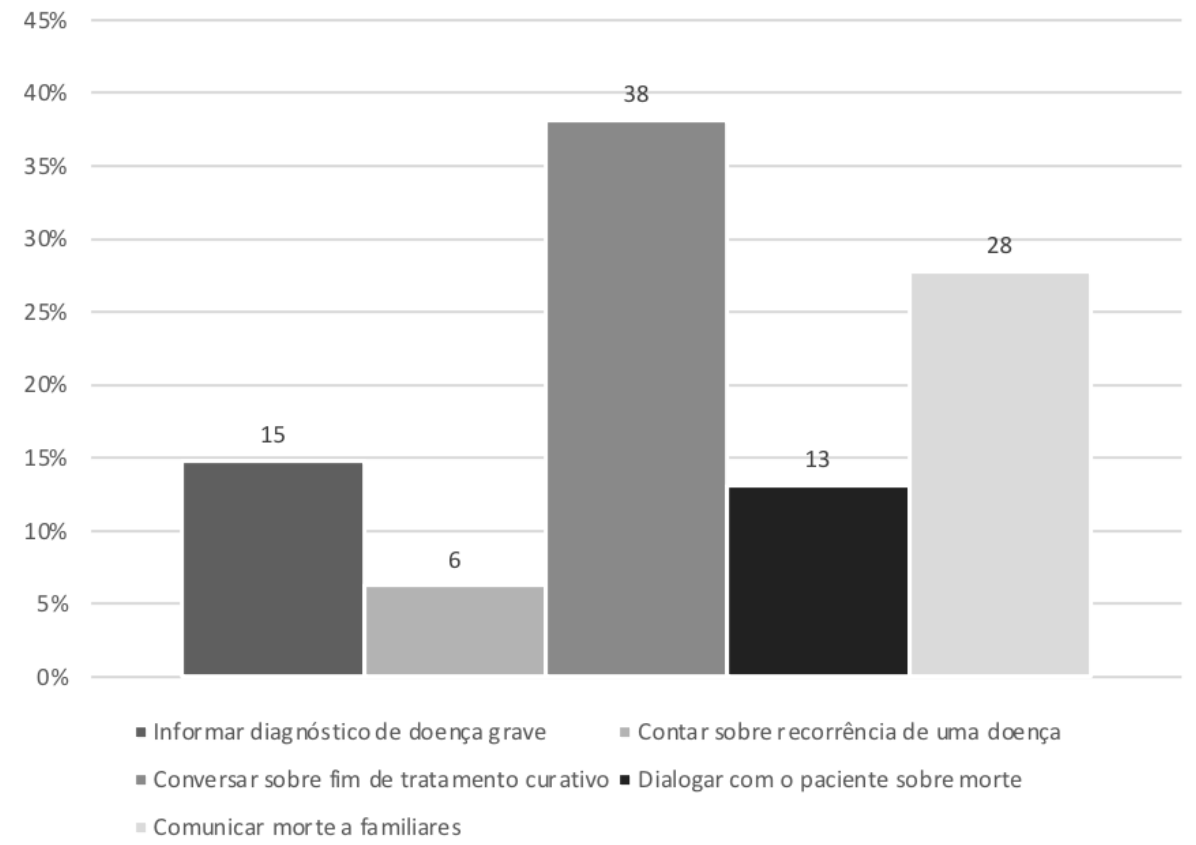

Ainda sobre dificuldades no processo de comunicar a má notícia, de forma mais específica em como manejá-la, que consiste na articulação das atividades com o objetivo de tomar decisões, os participantes consideram que mais difícil é ser honesto, sem tirar a esperança (69\%) e lidar com a emoção do paciente (59\%). No tocante aos processos de formação sobre como lidar com as emoções do paciente, numa consideração de que os participantes podiam marcar mais de um item, predominaram as aulas teóricas (41\%), nenhum treinamento (39\%) e a observação da prática de profissionais (38\%).

Além disso, os internos consideram que a comunicação, numa abordagem ampla, é a principal dificuldade vista na relação entre médico e paciente. Dentro disso, sugerem que a habilidade que está com maior deficiência no desenvolvimento da educação médica no local estudado, especialmente até o oitavo semestre, é lidar com as emoções que surgem no processo terapêutico, o que se relaciona com o tema de despreparo emocional para dar más notícias. 


\section{Gráfico 2 - Tipo de treinamento para lidar com as emoções do paciente}

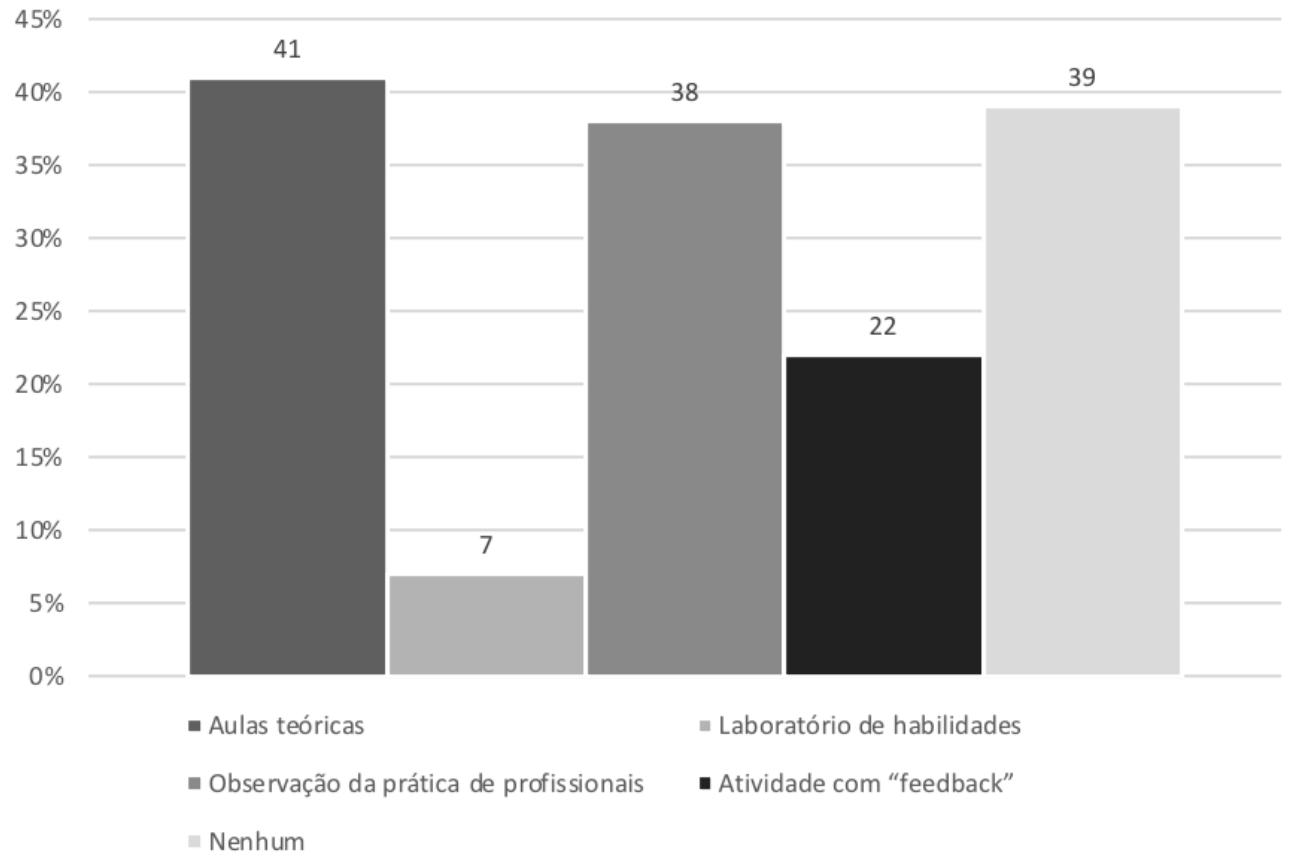

[...]até o S8 a gente não teve a experiência de atender muitos pacientes, então você fica desesperado, meu Deus, o que eu faço? O que eu digo? Socorro! (GF, L. I4)

Os participantes relatam a frequente terceirização das más notícias para lidar com essas dificuldades:

Os cuidados paliativos estão lá pra poder fornecer essa consulta e às vezes criar um paralelo com a família por ser uma equipe multidisciplinar [...] mas como muitas outras ocasiões, a gente terceiriza essas obrigações pra uma especialidade. (GF, D. I2)

Foi aplicada pergunta quanto à percepção dos estudantes sobre suas competências em contar más notícias e, na distribuição por semestre, e teve-se diferença ao longo do internato, com $61 \%$ do total de estudantes do I1 considerando sua habilidade ruim e $35 \%$ como boa. Já para os estudantes do $\mathrm{I} 4,78,6 \%$ a percebem como boa e $21,4 \%$ como ruim.

Quanto ao conhecimento sobre estratégias de interação com o paciente houve diferença relevante entre o primeiro e o quarto semestre do internato, indicando aprendizado quanto a isso. Do total de participantes que estava cursando o primeiro semestre, cerca de $40 \%$ têm conhecimento sobre as estratégias e 60\%, não. Do total de internos do quarto semestre, $59,5 \%$ têm conhecimento sobre as estratégias e 40,5\%, não.

Diante da frequente necessidade de comunicar más notícias, os internos procuram aprender através da experimentação, seja observando a conduta de professores e residentes, efetuando a comunicação, quando em conversas com os pacientes, ou se eximindo dessa atividade.

Eu percebo é que a gente não tem muito bem definido onde o interno se insere na comunicação de uma má notícia [...] vai se adaptando e fazendo como pode, né, ou até não fazendo. Tem gente que eu percebo que se afasta também, né, que sofre muito com essas coisas, que não consegue ficar nessa situação. (GF, K. I1)

O protocolo Spikes é conhecido por $99,4 \%$ dos participantes. No seu uso, as etapas consideradas mais fáceis foram preparar-se para o encontro com o paciente $(34,1 \%)$ e perceber o paciente $(18,7 \%)$.

Quanto aos elementos mais difíceis do protocolo Spikes teve-se o momento de expressão das emoções $(41,5 \%)$ e de transmissão das informações $(27,3 \%)$. 
Gráfico 3 - Etapa mais fácil do protocolo Spikes

$40 \%$

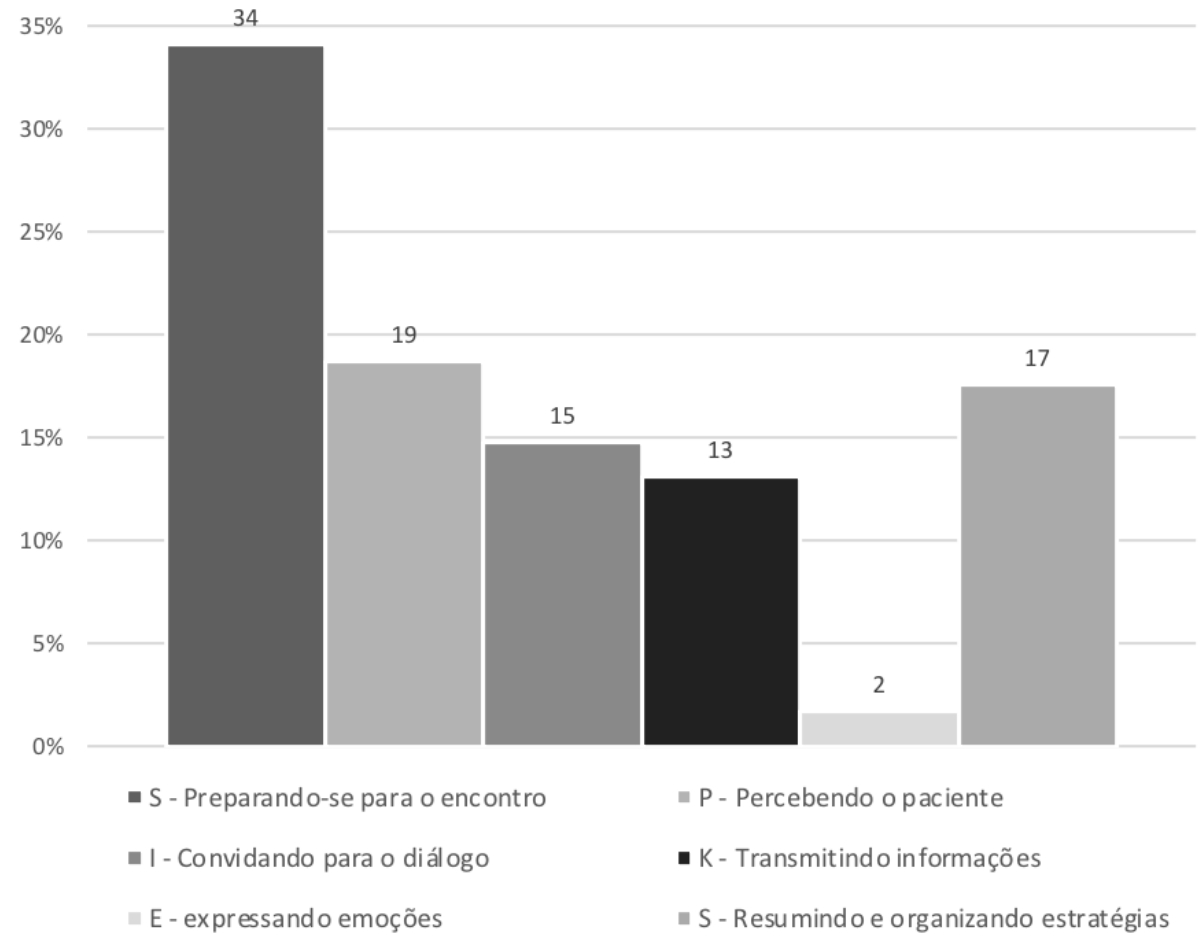

Gráfico $_{4}$ - Etapa mais difícil do protocolo Spikes

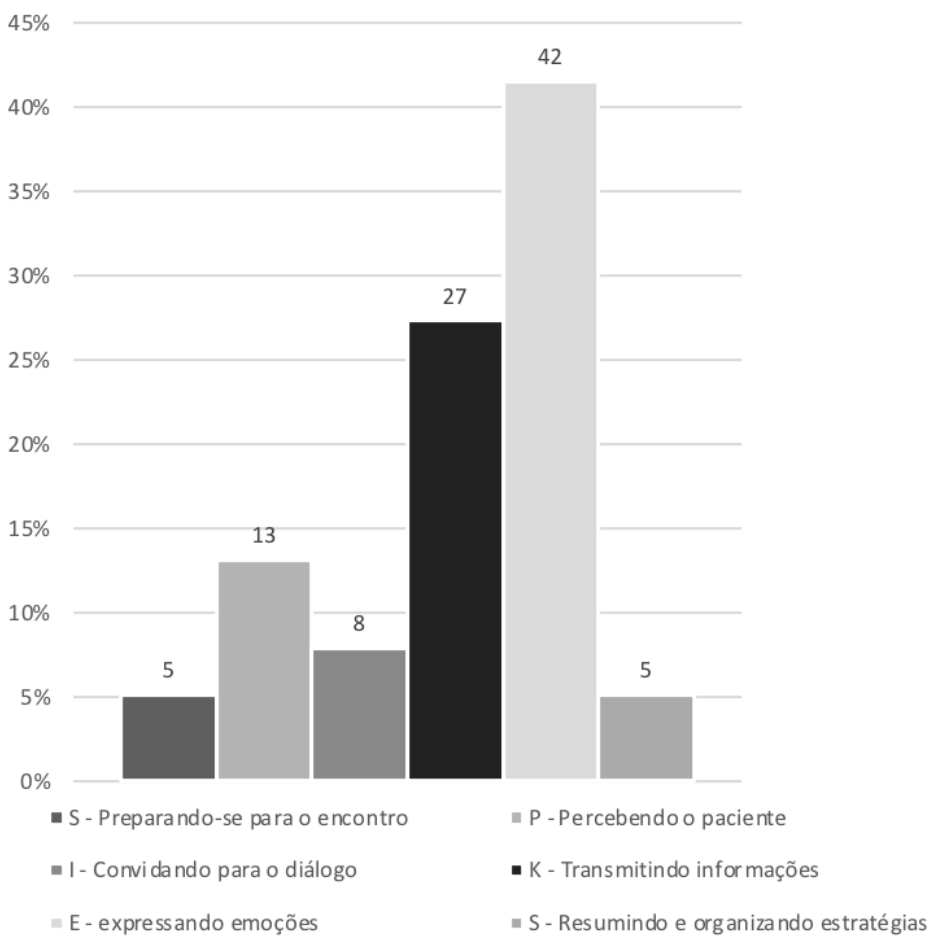


Viu-se que o protocolo Spikes é importante para as situações de comunicação, como apoio, seguindo seu passo a passo até que o processo de aprendizado se concretize e o protocolo possa ser aplicado sem rigidez. A fala a seguir traz elementos centrais do tema "percepção dos internos sobre o protocolo Spikes".

O Spikes é ótimo pra você usar como rodinha de bicicleta [...] A gente sempre tem noção que aquilo é engessado demais pra utilizar numa prática real, mas a gente sóvai conseguir [des] engessar e trazer aquilo ali pro paciente de uma forma mais fluida depois que se acostumar a fazer o engessado. (GF, C.I4)

Os participantes informaram ser incentivados, na formação em medicina, a realizar perguntas abertas para que o paciente possa se expressar (85\%); estabelecer metas e planos junto com o paciente $(75 \%)$, oferecer informações pausadamente $(68,2 \%)$; oferecer feedback após a manifestação de sentimentos $(57,4 \%)$; estimular a manifestação dos sentimentos (44,3\%) e $4 \%$ afirmaram não serem incentivados a realizar nenhuma dessas ações. Não foram encontradas diferenças relativas aos semestres.

Contudo, no tema sobre a educação médica para a má notícia, viu-se a necessidade de fomentar o processo de formação acerca da CMN para que os médicos não continuem com a sensação de despreparo quanto ao tema e, consequentemente, solicitem profissionais de outros serviços para essa ação. Além disso, que cuidem da relação médico-paciente no sentido informativo também, reforçando que, diferente disso, a relação está fadada ao fracasso.

Você se vê dentro de uma questão que o serviço tá utilizando os cuidados paliativos pra darem a notícia que eles não estavam dispostos a dar e que pra mim mostra que na verdade a relação médico/paciente era falida, porque será que é mais confortável pro meu paciente se comunicar com alguém que tá ao lado dele desde o início [...] ou com a equipe que chegou naquele dia e leu o prontuário dele? (GF, E. I1)

Apontam uma perspectiva de educação médica que deve ultrapassar a visão tecnicista, com ênfase no diagnóstico e tratamento, para chegar ao cuidado com as pessoas. Sugerem que o aprendizado sobre os aspectos relacionais deve abordar metodologias de ensino além das aulas teóricas, para que possibilitem a assimilação de processos de conexão e empatia, ressaltando o tema do aperfeiçoamento da competência comunicacional.

Medicina não é só diagnóstico difícil, complexo e tratamento. $\varepsilon$ isso éuma coisa que eu me estressava demais até chegar no internato, que eles querem que a gente saia um excelente médico, um médico completo e quando a gente sai [...] a gente não sabe nem se comunicar com a equipe. (GF JV, H.I3)

A gente passa seis anos aqui no mínimo, a gente tem que saber usar essa ferramenta, mas não sóo Spikes, aprender a se conectar com as pessoas e isso a gente não aprende. Tem uma aula, eu lembro que me revoltei com isso, que era uma aula sobre empatia com slides, oitenta pessoas na sala, ridículo, eu me revoltei. (GF, I.I2)

Na perspectiva de estabelecer maior conexão entre as pessoas, os participantes apontaram a empatia como um aspecto muito importante. Referem-se à empatia cognitiva, que possibilita maior entendimento do ponto de vista da outra pessoa, mas também destacaram a empatia emocional, como habilidade que possibilita a aproximação com o outro em sua inteireza.

Empatizaràs vezes parece uma coisa muito abstrata e como é que a gente empatiza? Acho que é o mínimo, é você olhar pra pessoa, olhar nos olhos como se tivesse conversando com qualquer outra pessoa que você já tem um vínculo, ouvir, observar aquela pessoa, o que ela traz de demanda pra você. (JV, J.I3)

Junto da empatia, foram elencados o conhecimento, a comunicação e a paciência, assim como outros aspectos que constituem a relação médico-paciente.

[...]às vezes só de você calar e deixar ele extravasar o sentimento dele e esperar passar pra depois falar alguma coisa mais técnica, combinar alguma coisa [...] acho que já é muita coisa [...] o paciente tem uma 
reação explosiva ou começa a chorar copiosamente, o importante évocêfazer o paciente sentir que você tá ali por ele [...] às vezes é você só deixar estar, oferecer um lenço, uma água e esperar.(GF, L.I4)

Além disso, os participantes destacam a importância do trabalho em equipe tanto no tocante à equipe de cuidados paliativos quanto ao trabalho da equipe assistencial. Esses dados evidenciam o tema nomeado de poder biomédico e a interdisciplinaridade na má notícia, mostrando a necessidade de transformações no sentido de compartilhamento de poder entre os membros da equipe para que seja possível a efetivação dos saberes das diversas disciplinas, que se revela a partir da prática dialógica das categorias profissionais. Nesse contexto, propõem que os profissionais façam considerações, entre si e com os internos, sobre suas atuações nos processos de trabalho.

Se a pessoa perguntar, também ter a humildade de dizer: olha, não sei, vamos ver aqui, vou chamar a assistente social, vou chamar a psicóloga, incluir a equipe. (GF, A. I3)

Eu tô fazendo direito? Eu acho que eu tô, mas será que eu não poderia melhorar? (GF, G.I2)

Os dados mostram diferenças significativas entre a postura de quem está no primeiro semestre do internato em relação a quem está no último semestre. Os participantes informaram ter dificuldades em relação ao tema da comunicação e que essa competência não é priorizada na formação, o que os fez sugerir o treinamento mais sistematizado dessa competência ao longo da graduação, com observação direcionada ao tema, prática com supervisão e feedback. Além disso, apontam para a necessidade do desenvolvimento da habilidade comunicacional, no tocante a lidar com as emoções, ao longo do processo do internato.

\section{Discussão}

Sabe-se que a comunicação de más notícias causa sensações desagradáveis em pelos menos um dos seus agentes e precisa ser aperfeiçoada para que haja real interação entre o tripé envolvido nessa ação: paciente-família-equipe de saúde. Ela pode ser terapêutica e contribuir positivamente para a vivência do processo de adoecimento, mas também pode ser nociva, comprometendo a saúde física e mental dos envolvidos (Santos et al., 2015; Monteiro; Quintana, 2016). Neste estudo, os participantes consideram que o processo de aprendizado da CMN evoluiu positivamente ao longo do internato, mas apresentam clareza das dificuldades.

Como descrito nos resultados, a tarefa considerada mais difícil, superando até mesmo a comunicação da morte de um paciente aos familiares, é conversar sobre o fim do tratamento, o que está em consonância com os dados de pesquisas como a de Vogel et al. (2019), evidenciando que a educação médica é voltada para a manutenção da vida e para a vitória sobre a doença. Contudo, eles não sabem como comunicar algo que pouco foi apresentado como uma possibilidade do cuidado médico, em virtude da frágil discussão sobre a morte.

Essa dificuldade tem relação com o que, no GF, os participantes nomearam de terceirização da notícia. Isso se efetiva na CMN primeiramente a um familiar, transferência considerada inadmissível na prática em saúde quando o paciente está consciente; ou no convite de uma equipe de cuidados paliativos para fazê-lo. Registrada a devida importância do trabalho especializado da equipe de cuidados paliativos percebe-se também a necessidade de fomentar a perspectiva de trabalho multidisciplinar, com consideração dos papéis profissionais como suporte ao indivíduo em seu processo saúde-doença e no fortalecimento da relação dessa equipe assistencial com o paciente e sua família (Back et al., 2007).

No manejo da CMN, emerge a necessidade de conjugação das "tecnologias leves" ao trabalho técnico. Os internos apontaram, como dificuldades mais recorrentes, a dificuldade em ser honesto sem tirar a esperança e lidar com as suas emoções e as dos pacientes, vistos também com Pereira (2017). Ser honesto sem tirar a esperança ou a vontade de viver dos pacientes faz parte das proposições do protocolo Spikes, em etapas como o diálogo sobre o conhecimento e o encaminhamento de estratégias com os pacientes e familiares. 
Informar a verdade ao paciente faz parte das obrigações legais do médico e, antes disso, apresentase como ação de consideração humanitária. Nessa direção, Hurst et al. (2015) enfatizam a importância de ensinar aos estudantes de medicina como se preparar para lidar com esse momento da CMN, escolhendo as palavras corretas, se adaptando à realidade do paciente, oferecendo suporte, expressando empatia.

Nesse contexto, o exercício da empatia tornase mais desafiador. Os estudos de Riess (2020) apontam que a empatia pode ser desenvolvida mesmo em quem, inicialmente, está desconectado ao outro. O desenvolvimento dá-se a partir de ações que demonstrem interesse, como prestar atenção em suas expressões. A partir disso, pode ocorrer a conexão entre as pessoas. Apesar de estudos consagrados como esse e de mudanças nas diretrizes curriculares da educação médica, sabe-se que as dificuldades de CMN apresentadas pelos médicos persistem e têm relação, também, com a formação. Poucas universidades valorizam o ensino da comunicação em seus currículos e é de suma importância o investimento em métodos que capacitem tais estudantes a desenvolver e aprimorar essa habilidade (Souza; Santos, 2013).

Esses elementos são vistos no trabalho das emoções dos estudantes de Medicina, numa perspectiva ampla. Neste estudo, 59\% dos participantes apontou ter dificuldade em lidar com as emoções e, no GF, informaram que essa é a habilidade com maior deficiência no desenvolvimento educacional, especialmente até o oitavo semestre. Saber lidar com as reações dos pacientes e com as próprias emoções e sentimentos é fundamental para o profissional que lida com a CMN, as quais têm o potencial para mudar muito a vida das pessoas.

Embora nas duas últimas décadas tenham ocorrido mudanças curriculares nos cursos de medicina com o objetivo de formar profissionais mais humanizados e capazes de atender as demandas atuais (Sombra Neto et al., 2017), ainda há muitos desafios quanto a isso, como a priorização dada ao tema ou mesmo a dinamicidade dos contextos. Com a vivência da pandemia de covid-19, em que há muitas pessoas afetadas pelo processo de adoecimento, pela morte e pelos lutos, evidencia-se ainda mais a importância de atuações médicas na CMN de maneira eficiente.

Uma boa comunicação nesses casos está relacionada a várias habilidades conjuntas para lidar com tais circunstâncias: comunicação não verbal, uma escuta atenta, falar a verdade com positividade, lidar com as emoções pessoais e familiares, respeitando o tempo necessário para recuperação do indivíduo (Liu et al., 2015). O treinamento desses aspectos comunicacionais, devido à sua complexidade, demanda tempo e repetição, o que se evidencia de maneira oposta nos dados deste estudo quanto aos processos de formação sobre como lidar com as emoções do paciente, em que predominaram as aulas teóricas (41\%), nenhum treinamento (39\%) e a observação da prática de profissionais (38\%)

Ao mesmo tempo, quando perguntados sobre incentivos gerais ao processo de $\mathrm{CMN}$ na educação médica, sem especificidade quanto às emoções, os internos informaram a priorização na realização de perguntas abertas para que o paciente possa se expressar (85\%); no estabelecimento de metas e planos junto ao paciente $(75 \%)$ e na oferta de informações de forma pausada $(68,2 \%)$. Estimular a manifestação de sentimentos e oferecer feedback quanto a eles estiveram presentes num percentual mais abaixo que os três citados anteriormente, coadunando com o que foi sugerido pelos participantes, no GF, como necessidade de incremento à formação: treinamento mais sistematizado da competência comunicacional ao longo dos semestres da graduação, com observação direcionada ao tema, prática com supervisão e retorno para os estudantes.

Interessante observar que o protocolo Spikes é conhecido por quase todos os participantes da pesquisa, 99,4\%. Isso é relevante, dada a sua utilidade e larga aplicação no mundo. A etapa mais fácil esteve na preparação para o encontro, que consiste na ambientação, numa preparação para uma comunicação que precisa informar e, especialmente, acolher. A maior dificuldade apontada pelos participantes quanto às etapas do protocolo foi o momento de expressão das emoções, deixando claro que esse é um dos principais desafios no processo de formação, o que tem sido observado em outros estudos, por ser a fase mais subjetiva e 
menos planejada, visto que a resposta do profissional deve ser de acordo com as emoções do paciente, na perspectiva de estabelecer apoio (Santos, 2017; Feitosa Junior et al., 2018).

Esse aspecto ficou mais evidenciado na pandemia de covid-19, contexto em que algumas dificuldades relativas à CMN são apresentadas com maior intensidade, dado que estudantes e profissionais estão submetidos a mais fatores estressores como risco aumentado de ser infectado, adoecer e morrer; sobrecarga e fadiga; exposição à morte em larga escala; frustração em não salvar vidas, apesar dos esforços, dentre outros (Schmidt et al., 2020).

Na vivência da pandemia, os estudos têm mostrado que os profissionais expostos diretamente aos riscos de contaminação, especialmente em hospitais e postos de saúde, registram, dentre outros aspectos, manifestação de sintomas de estresse agudo e redução da empatia (The Lancet, 2020b; Cruz et al., 2020). Além de ficarem isolados de suas famílias, testemunham ainda colegas de sua própria equipe indo a óbito. Segundo dados do Ministério da Saúde, o número de profissionais de saúde infectados com a covid-19 no Brasil mais do que dobrou entre maio e junho de 2020, contabilizando mais de 80 mil infectados e 169 mortes, o que colocou o país como líder nesse quesito (Lopes; Coletta, 2020).

Além disso, devido ao alto contágio, os familiares não podem acompanhar ou visitar seus entes queridos e a comunicação com os profissionais de saúde passa a ser preferencialmente de forma remota. Esses aspectos mostram que profissionais e estudantes estão desafiados a se adaptar, mais rapidamente, à realidade sanitária e à realidade do paciente, oferecendo suporte, expressando empatia, o que leva à necessidade de um redimensionamento da importância da relação entre os profissionais de saúde, paciente e família, incluindo o aperfeiçoamento na comunicação.

\section{Considerações finais}

Esta pesquisa foi concluída num momento importante de desenvolvimento da pandemia de covid-19, contexto em que a comunicação de más notícias esteve ainda mais presente, com muitos casos em que o tempo para assimilação e compreensão das informações foi insuficiente, dada a rápida evolução da doença e o prognóstico desfavorável, resultando em mortes.

A partir das diretrizes curriculares nacionais atuais, os projetos pedagógicos dos cursos de medicina brasileiros foram transformados, teoricamente, no que diz respeito à ênfase na comunicação. Contudo, foi observado um despreparo dos estudantes para lidar com a CMN, especialmente despreparo emocional. Isso leva a indicação de aprimoramento dos processos de educação médica, no sentido formal, e também sobre a necessidade de que os estudantes de Medicina sejam incentivados ao fomento do autoconhecimento.

Diante das dificuldades apresentadas, os internos participantes deste estudo apresentaramse críticos à condução de processos de formação focados no desenvolvimento técnico-científico. Demonstram encontrar caminhos complementares, com professores de referência, estudos e práticas que possibilitam o desenvolvimento de um olhar para o paciente que ultrapassa essa perspectiva, explicitando sensibilidade e a necessidade de que o cuidado seja priorizado, numa proposta de fortalecimento de relações empáticas que beneficiem o paciente, seus familiares e a própria equipe de saúde.

A avaliação dos resultados não pode deixar de considerar algumas limitações do estudo, como a fragilidade de utilização do grupo focal como instrumento central para obtenção dos dados dentro da abordagem qualitativa, considerando o risco de os participantes sentirem-se constrangidos em responder de acordo com o que é esperado socialmente, dado que estavam num grupo formado por seus futuros colegas de profissão.

Contudo, a pesquisa apresenta contribuições ao debate sobre o desempenho teórico-prático das CMN com ênfase nas impressões dos estudantes sobre a educação médica, na capacitação para lidar com as emoções, na percepção sobre um protocolo de referência no tema, nas necessidades identificadas e em sugestões para aperfeiçoar o desenvolvimento da competência comunicacional.

Os resultados deixaram evidente que a comunicação de más notícias não se limita à questão técnica, mas envolve sentimentos e atitudes que precisam ser abordados e trabalhados com metodologias diversas. Essas informações 
têm o potencial para contribuir com o fomento de discussões qualificadas sobre o assunto e, quiçá, incrementar a formação local e implementar políticas de educação na área médica.

\section{Referências}

BACK, A. L. et al. Efficacy of communication skills training for giving bad news and discussing transitions to palliative care. Archives of Internal Medicine, Chicago, v. 167, n. 5, p. 453-6o, 2007.

BARDIN L. Análise de conteúdo. Rio de Janeiro: Edições 70, 2011.

BARRETO, C. Estresse e isolamento: o "novo normal" de ser profissional de saúde durante a covid-19. Portal PebMed, Rio de Janeiro, 18 maio 2020.

BELLI, L. F. Recomendaciones para la comunicación de malas noticias por teléfono durante la pandemia por SAR-CoV-2. Comunicación breve. Revista Panamericana de Salud Pública, Washington, DC, v. 4, 2020.

BRASIL. Ministério da Educação. Resolução n. 3 de 20 de junho de 2014. Diretrizes curriculares nacionais do curso de graduação em medicina. Brasília, DF, 2014.

BRASIL. Ministério da Saúde. Plano de contingência nacional para infecção humana pelo novo coronavírus. Centro de Operações de Emergências em Saúde Pública - COE-COVID-19, Brasília, DF, 2020a.

BRASIL, Ministério da Saúde. Plataforma integrada de vigilância em saúde, Brasília, DF, $2020 b$.

CAMARGO, N C. et al. Ensino de comunicação de más notícias: revisão sistemática. Revista Bioética. Brasília, DF, v. 27, n. 2, p. 326-40. 2019. COVID-19 in Brazil: "So what?". The Lancet, Amsterdam, v. 395, n. 10235, p. 1461. 2020a. COVID-19: protecting health-care workers. The Lancet, Amsterdam, v. 395, p. 922. 2020b.

CRUZ, R. M. et al. Covid-19: emergência e impactos na saúde e no trabalho. Revista Psicologia:

Organizações e Trabalho. Florianópolis, v. 20, n. 2, p. 1-2, 2020.
FLICK, U. Introdução à pesquisa qualitativa. 3. ed. Porto Alegre: Artmed, 2009.

LIMA, B. Taxa de contágio da covid-19 volta a subir e doença está descontrolada. Correio Braziliense, 24 jun. 2020.

LIU, X. et al. Doctor-patient communication skills training in mainland China: a systematic review of the literature. Patient Education and Counseling. Amsterdam, v. 98, n. 1, p. 3-14, 2015.

LOPES, R.; COLETTA, R. D. Número de casos do coronavírus em profissionais de saúde mais do que dobra em um mês. Folha de S.Paulo, São Paulo, 12 jun. 2020.

MONTEIRO, D T; QUINTANA, A M. A comunicação de más notícias na UTI: perspectiva dos médicos. Psicologia: Teoria e Pesquisa, Brasília, DF, v. 32, n. 4, p. 1-9, 2016.

OLIVEIRA, M. C. Análise de estratégia para desenvolvimento de habilidade de comunicação de más notícias na formação de profissionais de medicina. 2018. 86f. Dissertação (Mestrado em Tecnologias em Saúde) - Escola Bahiana de Medicina e Saúde Pública. Salvador, 2018.

PEREIRA, C. R. Comunicando más notícias: protocolo PACIENTE. 2010. 10of. Tese (Doutorado em Anestesiologia) - Universidade Estadual Paulista - UNESP. São Paulo, 2010.

RIESS, H. Effetto empatia: le 7 chiavi delle neuroscienze per trasformare il nostro modo di amare, lavorare e comunicare. Trento: Edizioni Centro Studi Erickson; 2020.

SANTOS, A.L. P. Comunicação de más notícias: uma análise dessa prática entre médicos residentes. 2017· 34f. Monografia (Graduação em Medicina) - Universidade Federal do Maranhão. São Luís, 2017.

SANTOS, L F. et al. Quando a comunicação é nociva no encontro entre profissional e família da criança hospitalizada. Enfermería Global, Murcia, n. 37, p. 205-15, 2015.

SCHMIDT, B. et al. Saúde mental e intervenções psicológicas diante da pandemia do novo 
coronavírus (covid-19). Estudos de Psicologia. Campinas, v. 37, p. 1-13, 2020.

SOMBRA NETO, L L S. et al. Habilidade de comunicação da má notícia: o estudante de medicina está preparado? Revista Brasileira de Educação Médica, Brasília, DF, v. 41, n. 2, p. 26o-268, 2017. SOUZA, L V; SANTOS, M A. Quem é o especialista? Lugares ocupados por profissionais e pacientes no tratamento dos transtornos alimentares. Estudos de Psicologia, Campinas, v. 18, n. 2, p. 259-267. 2013.

STOVALL, M C. Oncology communication skills training: bringing science to the art of delivering bad news. Journal of the Advanced Practitioner in Oncology, Chicago, v. 6, n. 2, p. 162-166, 2015.
TANG, W R. et al. Truth telling in medical practice: students' opinions versus their observations of attending physicians' clinical practice. Psycho-oncology, Hoboken, v. 22, n. 7, p. 1605-1610, 2013.

TEIKE-LÜTHI F; C. B. Breaking bad news: "EPICES”, a French style as a learning method. Revue Médicale Suisse, Chêne-Bourg, v. 7, n. 277, p. 85-87, 2011.

UNIVERSIDADE FEDERAL DO CEARÁ. Faculdade de Medicina. Projeto pedagógico do curso de medicina 2018.1. Fortaleza, 2017.

VOGEL K. P. et al. Comunicação de más notícias: ferramenta essencial na graduação médica. Revista Brasileira de Educação Médica, Brasília, DF, v. 43, n. 1, p. 314-321, 2019.

\section{Contribuição dos autores}

Ribeiro e Batista contribuíram com a concepção do projeto e elaboração do desenho do manuscrito - introdução, metodologia, análise dos dados, resultados e redação final do manuscrito. Souza, Florêncio, Jorge e Raquel contribuíram com a análise dos dados e revisão crítica do conteúdo. Ribeiro colaborou com a aprovação da versão final do manuscrito.

Recebido: $30 / 03 / 2021$

Aprovado: 03/05/2021 